\title{
Obesity induces DNA damage
}

\author{
Constantin Stefani1 ${ }^{1,2}$, Alexandra Totan ${ }^{3}$, Daniela Miricescu ${ }^{3}$, \\ Ana Maria Alexandra Stanescu', Maria Greabu' ${ }^{1}$ \\ ${ }^{1}$ Department of Family Medicine, "Carol Davila" University of Medicine and Pharmacy, \\ Bucharest, Romania \\ 2"Carol Davila” University Central Emergency Military Hospital, Bucharest, Romania \\ ${ }^{3}$ Department of Biochemistry, Faculty of Dental Medicine "Carol Davila" University of Medicine and \\ Pharmacy, Bucharest, Romania
}

\begin{abstract}
A diet rich in saturated lipids, refined carbohydrates associated with a sedentary lifestyle leads to obesity in both adults and children. Obesity is associated with the existence of a chronic inflammatory process, that will further lead to systemic oxidative stress and DNA oxidative damage.

There is currently a positive correlation between obesity and cancer due to nuclear but also mitochondrial DNA injury. Obesity is a risk factor for the development of several types of cancer such as breast, prostate or colorectal.

The purpose of this review is to present the effects of obesity on human body, by describing the chronic inflammatory process, oxidative stress, and the molecular mechanisms involved in cancer progression.
\end{abstract}

Keywords: obesity, DNA damage, cancer

\section{INTRODUCTION}

Obesity is a serious health problem of the young but also of the adult population around the world. This complex chronic condition is characterized by significant increase in fat mass, disorders of lipid and glucose metabolism, chronic inflammation, oxidative stress (OS) and DNA damage. DNA damage has been reported in overweight or obese people, which will further lead to the development of cancer $(1,2)$.

USA has the highest rate of obesity, with more than $60 \%$ of the adult population being overweight or obese, followed by Europe. In USA after smoking, poor diet and lack of physical activity represent the second leading cause of mortality, causing 400,000 cases per year (3). A positive correlation has been reported between obesity and cancer incidence. In men, obesity is associated with an increased incidence of the esophagus, prostate, stomach, liver, pancreas, kidneys cancers. In women, a positive correlation was observed for breast, cervix, uterus and ovarian cancers (4).
Studies performed suggest that over 90,000 deaths annually recorded could be avoided if the adult population would have a normal weight and body mass index of less than 25 (4). In Romania, in 2018, according to the World Health Organization (WHO) lung, colorectal, breast, prostate and bladder the most common neoplasms for both sexes. For women from Romania, breast cancer has the highest incidence, and prostate cancer is the most common in men (5).

\section{OBESITY INDUCED INFLAMMATION AND OXIDATIVE STRESS}

In inflammation, neutrophils and macrophages are recruited and activated and will generate reactive oxygen (ROS) and nitrogen (RNS) species. Mitochondrial oxidative metabolism, apoptosis, enzymatic reactions of nicotinamide adenine dinucleotide phosphate (NAPPH) oxidases, superoxide dismutase, myeloperoxidase and nitric oxide synthase contribute to ROS production in cells (6). 
Adipose tissue secretes a large amount of pro-inflammatory cytokines such as IL- 6 and TNF- $\alpha$, IL6 , IL-10, MCP-1 (monocyte chemoattractant protein-1) which will lead to ROS (reactive oxygen production) production. IL- 6 and TNF- $\alpha$ activate C-reactive protein and again ROS production (7).

Obesity is associated with the installation of OS, due to the high and constant production of ROS and decreased levels of antioxidants. Increased levels of ROS lead to endogenous DNA damage. Lipids can undergo oxidation processes, stimulate the production of free radicals and the accumulation of these reactive species in the adipose tissue. Lipid peroxidation is induced by ROS and lead to the formation of DNA reactive lesions (7-9).

ROS are superoxide anion $\left(\mathrm{O}_{2}^{\circ}\right)\left(\mathrm{O}_{2}^{\circ}\right)$, hydrogen peroxide $\left(\mathrm{H}_{2} \mathrm{O}_{2} \mathrm{H}_{2} \mathrm{O}_{2}\right)$ and hydroxyl radical (HO) the most dangerous, being $\mathrm{HO}$, formed as a result of the Fenton and Weber-Weiss reactions. During aerobic respiration, $\mathrm{O}_{2}^{\circ} \mathrm{O}_{2}^{\circ}$ and $\mathrm{H}_{2} \mathrm{O}_{2} \mathrm{H}_{2} \mathrm{O}_{2}$ are generated. Neutrophils and macrophages activated in the inflammatory process generate numerous oxidizing agents such as peroxynitrite $\left(\mathrm{ONOO}^{-}\right.$, nitrosoperoxycarbonate $\left(\mathrm{ONOOCO}_{2}^{-}\right)$, hypochlorous acids $(\mathrm{HOCl})$ and diazot trioxide $\left(\mathrm{N}_{2} \mathrm{O}_{3}\right)(10)$.

\section{REACTIVE OXYGEN SPECIES AND DNA DAMAGE}

ROS participates in the process of lipid peroxidation with the formation of products such as malondialdehyde (MDA), which will interact with DNA forming DNA adducts (11).

ROS can also attack the nitrogenous bases from DNA structure, taking place processes of oxidation, methylation, nitration, deamination, double and single stranded DNA cleavage or cross-links in DNA structure. 8-hydroxy guanine (8-HOdG), 7,8 dihydroxy 8-oxoguanine, thymine glycol, 4,6-diamino 5-formamidopyrimidine (Fapy Ade) and 2,6-diamino-4-hydroxy-5-formamidopyrimidine (Fapy Gua) are the most common DNA mutations due to ROS attack. These DNA lesions can induce DNA mutations during replication. 8-HOdG being able to induce GC transversion to TA leading to activation of the mutagenesis process and cancer initiation $(12,13)$.

Adipose tissue via adipokines secrete proinflammatory cytokines such as TNF- $\alpha$ and IL- 6 that amplify the ROS formation process, which will contribute to DNA damage (14). Setayesh T et al. tested DNA damage in overweight mice and reported and increased level of injury versus the control group. Mice that received a diet poor in calories and protein, present about $30 \%$ decrease in DNA damage versus hypercaloric diet (15).

ROS may induce also lesions of mitochondrial DNA. Mitochondrial DNA is highly susceptible to oxidative damage because there are no nucleotide excision repair mechanisms (16).

Despite the fact that mitochondrial DNA encodes $1 \%$ of mitochondrial proteins, mitochondrial disorders are associated with an increased number of DNA lesions. Mitochondrial DNA damage is associated with diabetes, cancer and neurodegenerative disorders (16-18).

Obesity leads to beta oxidation process disorders and glucose homeostasis $(19,20)$. Mitochondrial DNA oxidation can induce the synthesis of pro-inflammatory cytokines such as IL- 6 , TNF- $\alpha$, pro-IL-1 $\beta$, through toll-like receptor activation (21).

\section{OBESITY AND CANCER}

Cancer is the leading cause of mortality in developing countries and the second leading cause in developing countries. 12.7 million cases and 7.6 million deaths were reported in USA in 2008 (22). In a report published in 2018 , the mortality rate from cancer is very high worldwide. Unfortunately, Romania occupies leading places in terms of lung, trachea, colorectal, breast and bronchus cancer (23).

The hormones released by adipokines, leptin and adiponectin are involved in cancer development. Leptin mediates tumor progression by activating AKT (protein kinase B), MAPK (mitogen-activated protein kinase) and STAT3 (signal transducer and activator of transcription proteins) signaling pathways. Adiponectin secreted especially by the visceral adipose tissue, has antitumor properties by activating AMPK (adenosine monophosphate-activated protein kinase) signaling pathways, the anti-inflammatory effects are manifested by inhibiting NFkB $(24,25)$.

Insulin and IGF-1 (insulin growth factor-1) are involved in the pathogenesis of cancer because it activates the AKT / mTOR signaling pathway, which promotes the growth and proliferation of tumor cells and inhibits cell survival (26).

AKT/mTOR signaling pathway suffers the most mutations in cancer, being a link between obesity and cancer (especially breast and colon) (26-29). Steroid hormones, namely estrogens, progesterone, androgens and adrenal steroids are associated with obesity and the development of certain types of neoplasms in women (30). 
Caloric restriction is associated with decreased cancer incidence, which will also lead to decreased AKT / mTOR activation. Tumors with activated $\mathrm{PI} 3 \mathrm{~K}$ are resistant to caloric restriction $(31,32)$. Caloric restriction reduces even the incidence of spontaneous tumor development in mice by $55 \%$ (22).

In a cohort study conducted by Alicja Wolk and coworkers included 28,129 patients (8165 men, 19964 women) who investigated the incidence of cancer in the period 1965-1993, according to the Swedish National Cancer Registry. The results of the study suggest that obese people have a 33\% incidence of cancer, $25 \%$ in men and $37 \%$ in women.

Increased incidence has been observed for the following types of cancer: small intestine, gallbladder, pancreas, larynx, bladder, uterus, cervix, endometrium, ovary connective tissue, Hodgkin's lymphoma (men) and non-Hodgkin's lymphoma in women (33).

In a study published in 2017 by Chen Y et al. analyzed 31 cohort studies to find an correlation between BMI and breast cancer incidence in postmenopausal or premenopausal women. Studies have shown a positive association between BMI and an increased incidence of breast cancer in postmenopausal women. No positive correlation was found in premenopausal European women between BMI and breast cancer (34).

A high fiber diet has beneficial effects on the body being involved in metabolism regulation, energy homeostasis and immune function. Fibers are involved in the fight against many disorders such as obesity, diabetes, dyslipidemia, hypertension or colon cancer (35).

\section{CONCLUSIONS}

The high consumption of hypercaloric foods leads to obesity. Chronic inflammation and OS induced by obesity cause DNA damage, which is involved in the pathogenesis of cancer. Caloric restriction and a diet rich in fruits and vegetables has beneficial effects on the human body and reduces the incidence of cancer.

\section{Acknowledgement}

All authors equally contributed to the present paper.

\section{REFERENCES}

1. Cerda C, Sanchez C, Climent B et al. Oxidative stress and DNA damage in obesity-related tumorigenesis. Adv Exp Med Biol 2014; 824: 5-17.

2. Zaki M, Basha W, El-Bassyouni HT et al. Evaluation of DNA damage profile in obese women and its association to risk of metabolic syndrome, polycystic ovary syndrome and recurrent preeclampsia. Genes Dis 2018; 5: 367-373

3. Mokdad AH, Marks JS, Stroup DF et al.Actual causes of death in the United States, 2000. Jama 2004; 291:1238-1245.

4. Calle EE, Rodriguez C, Walker-Thurmond K et al. Overweight, obesity, and mortality from cancer in a prospectively studied cohort of U.S. adults. N Engl J Med 2003; 24;348(17):1625-38.

5. International Agency for Research on Cancer. Romania Globocan 2018.

6. Dunn JD, Alvarez LA, Zhang X et al. Reactive oxygen species and mitochondria: A nexus of cellular homeostasis. Redox Biol 2015; 6:472-485

7. Vincent HK, Taylor AG. Biomarkers of potential mechanisms of obesity-induced oxidant stress in humans. Int J Obesty 2006; 30:400-418.

8. De Bont R, van Larebeke, N. Endogenous DNA damage in humans: A review of quantitative data. Mutagenesis 2004; 19:169-185.

9. Luczaj W, Skrzydlewska E. DNA damage caused by lipid peroxidation products. Cell Mol Biol Lett 2003; 8: 391-413.

10. Thomas C, Mackey MM, Diaz AA et al. Hydroxyl radical is produced via the Fenton reaction in submitochondrial particles under oxidative stress: Implications for diseases associated with iron accumulation. Redox Rep 2009: 14:102-108.

11. Piscitello D, Varshney D, Lilla $S$ et al. AKT overactivation can suppress DNA repair via p70S6 kinase-dependent downregulation of MRE11. Oncogene 2018; 37: 427-438.

12. Kamiya $\mathrm{H}$. Mutagenic potentials of damaged nucleic acids produced by reactive oxygen/nitrogen species: Approaches using synthetic oligonucleotides and nucleotides: Survey and summary. Nucleic Acids Res 2003; 31: 517-531.

13. Sova H, Jukkola-Vuorinen A, Puistola U et al. 8-Hydroxydeoxyguanosine: A new potential independent prognostic factor in breast cancer. Br J Cancer 2010:102:1018-1023.

14. Arango Duque G, Descoteaux A. Macrophagecytokines: Involvement in immunity and infectious diseases. Front Immunol 2014; 5: 491.

15. Setayesh T, Mišík M, Langie SAS et al. Impact of Weight Loss Strategies on Obesity-Induced DNA Damage. Mol Nutr Food Res 2019; 63(17): e1900045.

16. Stein A, Sia EA. Mitochondrial DNA repair and damage tolerance. Front Biosci 2017; 22: 920-943.

17. Wang J, Xiong S, Xie $\mathrm{C}$ et al. Increased oxidative damage in nuclear and mitochondrial DNA in Alzheimer's disease. J Neurochem 2005; 93: 953-962.

18. Bensch KG, Mott JL, Chang SW et al. Selective mtDNA mutation accumulation results in beta-cell apoptosis and diabetes development Am J Physiol Endocrinol Metab 2009; 296: E672-E680.

19. Sutherland LN, Capozzi LC, Turchinsky NJ et al. Time course of high-fatdiet-induced reductions in adipose tissue mitochondrial proteins: Potential mechanisms and the relationship to glucose intolerance. Am J Physiol Endocrinol Metab 2008; 295: E1076-E1083.

20. Gao CL, Zhu C, Zhao YP et al. Mitochondrial dysfunction is induced by high levels of glucose and free fatty acids in 3T3-L1 adipocytes. Mol Cell Endocrinol 2010; 320: 25-33.

21. Shimada K, Crother TR, Karlin J et al. Oxidized mitochondrial DNA activates the NLRP3 inflammasome during apoptosis. Immunity 2012: 36: $401-414$ 
22. Siegel RD, Naishadham AJ. Cancer statistics, 2012. CA Cancer J Clin 2012; 62:10-29.

23. Cancer statistics-specific cancers. Eurostat, Statistics Explaned, 2018

24. Dalamaga M, Diakopoulos KN Mantzoros CS. The role of adiponectin in cancer: A review of current evidence. Endocr Rev 2012; 33: 547-594.

25. Gao J, Tian J, Lv Y et al. Leptin induces functional activation of cyclooxygenase-2 through JAK2/STAT3, MAPK/ERK, and PI3K/AKT pathways in human endometrial cancer cells. Cancer Sci 2009; 100: 389-395.

26. Renehan AG, Frystyk J, Flyvbjerg A. Obesity and cancer risk: The role of the insulin-IGF axis. Trends Endocrinol Metab 2006;17: 328-336.

27. Bonin S, Pracella D, Barbazza R et al. PI3K/AKT Signaling in Breast Cancer Molecular Subtyping and Lymph Node Involvement. Dis Markers 2019;2019:7832376.

28. Kim H, Ju JH, Son S et al. Silencing of CD133 inhibits GLUT1-mediated glucose transport through downregulation of the HER3/Akt/mTOR pathway in colon cancer. FEBS Lett 2019; : 10.1002/1873-3468.

29. Moore T, Beltran L, Carbajal S et al. Dietary energy balance modulates signaling through the Akt/Mammalian target of Rapamycin pathways in multiple epithelial tissues. Cancer Prev Res 2008; 1:65-76.

30. Kaaks R, Lukanova A, Kurzer MS. Obesity, endogenous hormones, and endometrial cancer risk: A synthetic review. Cancer Epidemiol Biomarkers Prev 2002; 11:1531-1543.

31. Kalaany NY, Sabatini DM. Tumours with PI3K activation are resistant to dietary restriction. Nature 2009; 458:725-731.

32. Jiang W, Zhu Z, Thompson HJ. Dietary energy restriction modulates the activity of Akt and Mtor in mammary carcinomas, mammary gland, and liver. Cancer Res 2008; 68: 5492-5499.

33. Wolk A, Gridley G, Svensson M et al. A prospective study of obesity and cancer risk (Sweden). Cancer Causes and Control 2001; 12:13-21.

34. Chen Y, Liu L, Zhou Q et al. Body mass index had different effects on premenopausal and postmenopausal breast cancer risks: A dose-response meta-analysis with $3,318,796$ subjects from 31 cohort studies. BMC Public Health 2018;17(1):936.

35. Mandaliya D, Patel S, Seshad S. Fiber in Our Diet and Its Role in Health and Disease. Functional Food and Human Health, 2018; 247-255. 